\title{
RATIONAL INNER FUNCTIONS ON BOUNDED SYMMETRIC DOMAINS
}

BY

\author{
ADAM KORÁNYI AND STEPHEN VÁGI
}

\begin{abstract}
It is shown that the rational inner functions on any bounded symmetric domain are given by a generalized version of a formula found by Rudin and Stout in the case of the polydisc. In particular, it is shown that all rational inner functions are constant on symmetric domains which have no irreducible factor of tube type.
\end{abstract}

1. Introduction. The rational inner functions on the unit polydisc $U^{n}$ of $\mathbf{C}^{n}$ are the functions of the form

$$
F(z)=M(z) Q^{\#}(r(z)) / Q(z)
$$

where $M$ is a monomial, $r\left(z_{1}, \ldots, z_{n}\right)=\left(z_{1}^{-1}, \ldots, z_{n}^{-1}\right), Q$ is a polynomial such that $Q(z) \neq 0$ for $z \in U^{n}$, and $Q^{\#}$ is obtained from $Q$ by conjugating coefficients. For $n=1$ this is a familiar elementary fact (the right-hand side of (1.1) is easily seen to be a finite Blaschke product). For $n>1$ the result is much more subtle, and is due to Rudin and Stout [15]. Rudin in [14] also proved that an inner function which is continuous on the closure $\overline{U^{n}}$ of $U^{n}$ must be a rational function with $Q(z) \neq 0$ for $z \in \overline{U^{n}}$. On the other hand, it is well known that an inner function on the unit ball $B^{n}$ of $\mathbf{C}^{n}$ which is continuous on $\overline{B^{n}}$ reduces to a constant, and it is also known that the same holds for rational inner functions.

The polydisc is the simplest bounded symmetric domain which is of tube type, while the ball is the simplest such domain which has no irreducible factor of tube type (see $\$ 2$ for definitions). In this paper we extend these results to all bounded symmetric domains. In Theorem 3.3 we prove that on the domains of tube type the rational inner functions, mutatis mutandis, are of the form (1.1), and that they reduce to constants on those domains which have no irreducible factors of tube type. The case of domains which have irreducible factors of both types can be reduced to the above two cases. In Proposition 3.4 we show that inner functions on a bounded symmetric domain $D$ which are restrictions to $D$ of (a) continuous functions on $\bar{D}$, or (b) meromorphic functions defined on domains containing $\bar{D}$, are rational. The basic results on bounded symmetric domains on which the proofs of the

Received by the editors September 29, 1978.

AMS (MOS) subject classifications (1970). Primary 32M15; Secondary 34B25. 
above statements hinge are Lemmas 2.3 and 2.4 in which, roughly, we construct the analogues of $M$ and $r$ in (1.1) for arbitrary domains of tube type. Once this is accomplished, we determine the polynomial inner functions in Lemma 2.5 by using results of Bochner [3]. Then the methods of Rudin and Stout with suitable modifications yield Theorem 3.3 and Proposition 3.4.

We thank Professor E. L. Stout for bringing reference [18] to our attention. Work on this paper was partially supported by grants from the N.S.F., MCS76-06960 and MCS77-03613.

2. Preparatory lemmas. In this section we have to rely substantially on results of [10] whose notation we shall follow quite closely. We begin by summarizing some general facts and definitions about the domains and the results needed from [10] for domains of tube type. Let $D$ be a bounded symmetric domain in $\mathbf{C}^{n}$ given in the Harish-Chandra realization [7, Chapter VIII], $D$ contains the origin 0 of $\mathbf{C}^{n}$. Let $G^{0}$ be the connected group of holomorphic automorphisms of $D, K$ the isotropy group of 0 in $G^{0}$. The group $K$ is connected and is a maximal compact subgroup of $G^{0}$ and acts on $\mathbf{C}^{n}$ by complex linear transformations, so does its complexification $K^{\mathbf{C}}$. Let $S$ be the Bergman-Šilov boundary of $D ; S$ is a compact real analytic submanifold of $\mathbf{C}^{n}$ to which the action of $G^{0}$ extends, and $K$ acts transitively on $S$. The center of $K$ contains a one-dimensional closed subgroup which acts on $\mathbf{C}^{n}$ via multiplication by complex numbers of modulus one, hence, $D$ and $S$ are circular sets. The domain always has a realization as a generalized half-plane, and $D$ is said to be of tube type if its half-plane realization is a tube domain over a homogeneous selfadjoint cone. This is the case if and only if $\operatorname{dim}_{\mathrm{R}} S=\operatorname{dim}_{\mathrm{C}} D$. The cartesian product of two bounded symmetric domains is also bounded symmetric, and its Silov boundary is the product of the Šilov boundaries of the factors. Every such domain $D$ is uniquely the product of irreducible factors $D_{j}$; it is of tube type if and only if each $D_{j}$ is. The group $G^{0}$ is the direct product of simple Lie groups $G_{j}^{0}$ which correspond to the $D_{j}$ 's. Similarly, $K$ is the direct product of groups $K_{j}$ which have one-dimensional center. In this section we deal only with domains of tube type, therefore, the subspaces $\mathfrak{f}_{2}, \mathfrak{l}_{2}, \mathfrak{q}_{2}$, and $\mathfrak{p}_{2}$ of $g^{\mathbf{C}}$ (the complexification of the Lie algebra $\mathfrak{g}^{0}$ of $G^{0}$ ) as defined in [10], are zero, and we shall omit without danger of confusion all subscripts 1 from $\mathfrak{f}_{1}, \mathfrak{l}_{1}, \mathfrak{q}_{1}$, and $\mathfrak{p}_{1}$. The decomposition $\mathrm{g}^{0}=\mathrm{f}+i \mathfrak{p}$ is a Cartan decomposition of $\mathrm{g}^{0}$. The complexification of $\mathfrak{p}$ splits as $\mathfrak{p}^{+}+\mathfrak{p}^{-}$, the respective spans of the positive and negative root spaces of the Cartan subalgebra $\mathfrak{h}^{\mathbf{C}}$ of $\mathfrak{g}^{\mathbf{C}}$, where $\mathfrak{h} \subset \mathfrak{t}$ is a Cartan subalgebra of $\mathfrak{g}^{\mathbf{0}}$. The set of Harish-Chandra's strongly orthogonal $\mathfrak{h}^{\mathbf{C}}$ roots is denoted by $\Delta$; to each $\alpha \in \Delta$ there corresponds a three-dimensional simple Lie algebra spanned by $E_{\alpha}, E_{-\alpha}, H_{\alpha}$ with $E_{ \pm \alpha} \in \mathfrak{p}^{ \pm}, H_{\alpha} \in i \mathfrak{h}$, and

$$
\left[H_{\alpha}, E_{ \pm \alpha}\right]= \pm 2 E_{ \pm \alpha}, \quad\left[E_{\alpha}, E_{-\alpha}\right]=H_{\alpha} \text {. }
$$


Set $\mathfrak{a}^{-}=\sum_{\alpha \in \Delta} \mathbf{R} E_{-\alpha}$, then $D$ is realized as a bounded domain in $\mathfrak{p}^{-}$(this is the Harish-Chandra realization). The groups $K$ and $K^{\mathrm{C}}$ act on $\mathfrak{p}^{-}$by the adjoint representation and

$$
D=\operatorname{Ad}(K)\left(D \cap a^{-}\right)=\operatorname{Ad}(K)\left\{\sum_{\alpha \in \Delta} \xi_{\alpha} E_{-\alpha}: \xi_{\alpha} \in \mathbf{R},\left|\xi_{\alpha}\right|<1\right\} .
$$

The Bergman-Šilov boundary $S$ is the orbit of $i E_{-\Delta}=i \Sigma_{\alpha \in \Delta} E_{-\alpha}$ (and, hence, also of $E_{-\Delta}$ ) under $K$; it is a compact symmetric space whose isotropy group at $i E_{-\Delta}$ is $L$. The corresponding Cartan decomposition is $\mathfrak{f}=\mathfrak{l}+\mathfrak{q}$, and $\mathfrak{h}^{-}=\sum_{\alpha \in \Delta} \mathbf{R} i H_{\alpha} \subset \mathfrak{q}$ is a Cartan subalgebra of this symmetric space. The noncompact dual symmetric space of $S$ is $K^{*} / L^{0}$ ( $L^{0}$ is the identity component of $L$ ) and arises as the orbit $\Omega$ of $E_{-\Delta}$ under $K^{*}=\exp t^{*}=$ $\exp (\mathfrak{l}+i q)$, and is contained in the real form $\mathfrak{n}^{-}$of $\mathfrak{p}^{-}$. The orbit $\Omega$ is a convex regular cone which is selfadjoint relative to the inner product $\langle,\rangle_{\nu}$. The half-plane realization $D^{c}$ of $D$ is the tube domain $\mathfrak{n}^{-}+i \Omega$ in $\mathfrak{p}^{-}$.

LEMma 2.1. The map $\psi: q^{\mathrm{C}} \rightarrow \mathfrak{p}^{-}$defined by $\psi(X)=\left[E_{-\Delta}, X\right] / 2$ is an $\operatorname{Ad}\left(L^{0}\right)$-equivariant complex vector space isomorphism such that $\psi(i q)=\mathfrak{n}^{-}$, and $\psi\left(H_{\alpha}\right)=E_{-\alpha}(\alpha \in \Delta)$.

Proof. The map $\psi$ is clearly complex linear, and $\psi\left(H_{\alpha}\right)=E_{-\alpha}$ by the strong orthogonality of $\Delta$ and by (2.1). Hence, $\psi$ carries $i \mathfrak{h}^{-}$onto $\mathrm{a}^{-}$which is contained in $\mathfrak{n}^{-}$[10, Proposition 6.2]. Since $\operatorname{Ad}\left(L^{9}\right)$ fixes $E_{-\Delta}, \psi$ is $\operatorname{Ad}\left(L^{9}\right)$ equivariant. Since $i \mathfrak{h}^{-}$is a Cartan subalgebra of the symmetric space $K^{*} / L^{0}$, and since $\operatorname{Ad}\left(L^{0}\right)$ preserves $\mathfrak{n}^{-}$, it follows that $\psi(i q) \subset \mathfrak{n}^{-}$. To see that $\psi$ is injective let $X=\operatorname{Ad}(l) \sum_{\alpha \in \Delta} c_{\alpha} H_{\alpha}$ with $l \in L^{0}, c_{\alpha} \in \mathbf{C}$ be an element of $q^{\mathbf{C}}$ such that $\psi(X)=0$. Then $\psi\left(\Sigma_{\alpha \in \Delta} c_{\alpha} H_{\alpha}\right)=\Sigma_{\alpha \in \Delta} c_{\alpha} E_{-\alpha}=0$ because $\operatorname{Ad}(l)$ is nonsingular and, hence, $c_{\alpha}=0, \alpha \in \Delta$. By Lemma 4.7 of [10] $i q$ and $\mathfrak{n}^{-}$ have the same dimension, hence, $\psi$ maps $i q$ onto $\mathfrak{n}^{-}$. Now $i q$ and $\mathfrak{n}^{-}$are respectively real forms of $q^{\mathbf{C}}$ and $\mathfrak{p}^{-}$, hence, $\psi$ maps $q^{\mathbf{C}}$ isomorphically onto $\mathfrak{p}^{-}$.

Lemma 2.2. Let $D$ be irreducible. Then the Weyl group of the pair ( $\left.f^{*}, i q\right)$ operates on $i \mathfrak{h}^{-}$by all permutations of the $H_{\alpha}$ 's $(\alpha \in \Delta)$.

Proof. It is known [12] that those restricted $i \mathfrak{h}^{-}$roots $\lambda$ of $\mathrm{g}^{\mathbf{C}}$ for which $\mathrm{g}^{\lambda} \in \mathrm{f}^{\mathrm{C}}$ are exactly those of the form $(\alpha-\beta) / 2$ with $\alpha, \beta \in \Delta$. These are the roots of the symmetric space $K^{*} / L^{0}$. (They span only a subspace of codimension one in $i \mathfrak{h}^{-}$, because $\mathfrak{f}^{*}$ is only reductive, not semisimple: $\mathfrak{f}^{*}=\left[\mathfrak{f}^{*}, \mathfrak{t}^{*}\right]$ $+\mathbf{R} Z^{0}[10$, p. 282].) This set of roots is a simple system of roots and so it determines the Killing form of $\mathfrak{f}^{*}$ up to a multiplicative constant. It follows that on $i \mathfrak{h}^{-}$the Killing form of $\mathfrak{f}^{*}$ is a multiple of the Killing form of $g^{\mathbf{C}}$ and, therefore, the Weyl group reflections can be calculated by using the Killing form of $g^{\mathrm{C}}$. It is known that for this form the $H_{\alpha}$ 's $(\alpha \in \Delta)$ are orthogonal and 
have the same length. It follows by computation that the reflection in $(\alpha-\beta) / 2$ carries $H_{\alpha}$ to $H_{\beta}$.

REMARK. The system $\{(\alpha-\beta) / 2\}_{\alpha, \beta \in \Delta}$ is of type $A$. This shows that a homogeneous selfadjoint cone has its restricted root system of type $A$. The converse is also true by the classification of symmetric spaces. This fact was previously proved by $\mathrm{O}$. Loos [11, Vol. 11, p. 171] by using Jordan algebras.

LEMMA 2.3. Let $D$ be a bounded symmetric domain of tube type: Then there exists a unique $L^{0}$-invariant polynomial $N$ on $\mathfrak{p}^{-}$whose restriction to $\mathfrak{a}^{-}$is given by $N\left(\sum_{\alpha \in \Delta} \xi_{\alpha} E_{-\alpha}\right)=\prod_{\alpha \in \Delta} \xi_{\alpha} . N$ is homogeneous, real on $\mathfrak{n}^{-}$and of absolute value one on $S$. Moreover, if $D$ is irreducible, then so is $N$. If the irreducible factors of $D$ are $D_{j}, j=1,2, \ldots, p$, and $N_{j}$ is the polynomial defined above corresponding to $D_{j}$, then $N=N_{1} \otimes N_{2} \otimes \cdots \otimes N_{p}$.

Proof. Consider on $i \mathfrak{h}^{-}$the polynomial function $\sum_{\alpha \in \Delta} \xi_{\alpha} H_{\alpha} \mapsto \Pi_{\alpha \in \Delta} \xi_{\alpha}$. This polynomial by Lemma 2.2 is invariant under the Weyl group of $K^{*} / L^{0}$. Therefore, by a theorem of Chevalley [7, p. 430] it extends uniquely to an $L^{0}$ invariant polynomial $N_{\mathrm{q}}$ on $\mathrm{q}^{\mathrm{C}}$. (Actually, in [7] the theorem is only proved for semisimple $K^{*}$, but it is easy to verify that it remains true for reductive groups which are the direct product of groups with one-dimensional center.) Set $N=N_{\mathrm{q}} \circ \psi^{-1}$, then by Lemma $2.1, N$ is an $\operatorname{Ad}\left(L^{9}\right)$-invariant polynomial on $\mathfrak{p}^{-}$which is equal to $\Pi_{\alpha \in \Delta} \xi_{\alpha}$ on $\mathfrak{a}^{-}$. This polynomial is unique because if $N^{\prime}$ is another such polynomial, then $N^{\prime} \circ \psi$ on $i \mathfrak{h}^{-}$coincides with $N_{\mathrm{a}}$ and hence is equal to $N_{\mathrm{q}}$ by the uniqueness assertion of Chevalley's theorem. On $\mathfrak{n}^{-}, N$ is real because it is real on $\mathfrak{a}^{-}$and $\mathfrak{n}^{-}=\operatorname{Ad}\left(L^{0}\right) \mathfrak{a}^{-}$, and because $\operatorname{Ad}\left(L^{0}\right)$ acts on $\mathfrak{n}^{-}$by real linear transformations [10, Proposition 6.2]. Since on

$$
\left(\mathfrak{a}^{-}\right)^{\mathbf{C}} \cap S=\left\{\sum_{\alpha \in \Delta} \xi_{\alpha} E_{-\alpha}: \xi_{\alpha} \in \mathbf{C},\left|\xi_{\alpha}\right|=1\right\}
$$

$N$ is of absolute value one, it is also of absolute value one on all of $S=\operatorname{Ad}\left(L^{g}\right)\left(\left(a^{-}\right)^{\mathrm{C}} \cap S\right)$ by $\operatorname{Ad}\left(L^{9}\right)$-invariance. Now suppose that $D$ is irreducible. Let $N=P_{1}^{m_{1}} \cdots P_{p}^{m_{p}}$ be a factorization of $N$ into irreducible factors. Since $N \circ \operatorname{Ad}(l)=N$ for $l \in L^{0}$, we have $P_{j} \circ \operatorname{Ad}(l)=c_{j}(l) P_{j^{\prime}}$ for some $j^{\prime}$ with $c_{j}(l) \in \mathrm{C}$. Continuous dependence on $l$ gives $P_{j} \circ \operatorname{Ad}(l)=$ $c_{j}(l) P_{j}$, and it follows immediately that $l \mapsto c_{j}(l)$ is a one-dimensional representation of $L^{0}$. Now let $K_{1}^{*}$ be $K^{*}$ modulo its center (which is the group of dilations of the irreducible factors of $\Omega$ ). By the remark following Lemma 2.2, $K_{1}^{*} / L^{0}$ is a product of symmetric spaces with restricted root system of type $A$, such spaces are never Hermitian symmetric [12], and hence $L^{0}$ is semisimple and, therefore, $c_{j}(l)=1$ for all $j=1,2, \ldots, p$. It follows that each $P_{j}$ is $\operatorname{Ad}\left(L^{0}\right)$-invariant and, therefore, there is a corresponding Weyl group- 
invariant factoring of $\left.N\right|_{\left(a^{-}\right)}$c. But by Lemma 2.2 there is no nontrivial factoring of this nature. The last statement of the lemma follows from the fact that $L^{0}$ is the direct product of the corresponding $L_{j}^{0}$ s.

REMARKS. (1) The Koecher norm function of $D$ (which is usually denoted by $N$ in papers dealing with these domains) is the $(n / l)$ th power of the present $N(n=\operatorname{dim} D, l=\operatorname{rank} D)$. The present $N$ coincides with the norm $H N$ used in [4].

(2) $N$ can be easily computed in every specific case. For the domains of square matrices and symmetric matrices it is the determinant. For the skew symmetric matrix domains it is the Pfaffian; for the $n$-dimensional Lie sphere it is $\sum_{j=1}^{n} z_{j}^{2}$. For the 27-dimensional exceptional domain realized in the space of $3 \times 3$ Hermitian matrices over the Cayley numbers, it is the Freudenthal determinant [6].

Let $D$ continue to be a bounded symmetric domain of tube type. In the following $z \mapsto \bar{z}$ will denote complex conjugation on $\mathfrak{p}^{-}$with respect to the real form $\mathfrak{n}^{-}$, and grad will denote the gradient of a function on $\mathfrak{n}^{-}$with respect to the inner product $\langle,\rangle_{\nu}$; in case of a holomorphic function grad will be extended to $\mathfrak{p}^{-}$by analyticity.

For all $z \in \mathfrak{p}^{-}$such that $N(z) \neq 0$ we define $r(z)$ by

$$
r(z)=(\operatorname{grad} N(z)) / N(z)
$$

LeMMa 2.4. For all $z \in S, \bar{z}=r(z)$.

Proof. By the last assertion of Lemma 2.3 it is enough to consider an irreducible $D$. By the Cartan decomposition every $z \in S$ is of the form

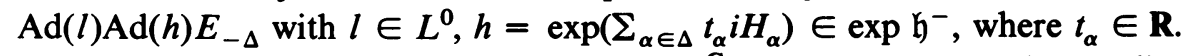
Assume first that $l$ is the identity, so $z \in\left(a^{-}\right)^{\mathbf{C}}$. Then by strong orthogonality of $\Delta$,

$$
z=\operatorname{Ad}(h) E_{-\Delta}=\sum_{\alpha \in \Delta} e^{i t_{\alpha}} E_{-\alpha}
$$

and since $E_{-\alpha} \in \mathfrak{n}^{-}\left[10\right.$, p. 282], $\bar{z}=\sum_{\alpha \in \Delta} e^{-i t_{\alpha}} E_{-\alpha}$. This shows that on $\left(a^{-}\right)^{\mathbf{C}} \cap S$ the map $z \rightarrow \bar{z}$ coincides with the restriction of the map $\sum_{\alpha \in \Delta} \xi_{\alpha} E_{-\alpha} \mapsto \Sigma_{\alpha \in \Delta} \xi_{\alpha}^{-1} E_{-\alpha}$ of $\left(\mathfrak{a}^{-}\right)^{\mathbf{C}}$, which can also be written as

$$
z \rightarrow\left(\operatorname{grad}_{\mathfrak{a}}-N(z)\right) / N(z)
$$

Here $\operatorname{grad}_{a^{-}}$is the gradient with respect to $\mathfrak{a}^{-}$, extended by analyticity to $\left(a^{-}\right)^{\mathbf{C}}$.

Next we shall show that at every point $z \in \mathfrak{a}^{-}$the gradient of $N$ relative to $a^{-}$coincides with its gradient relative to $\mathfrak{n}^{-} \cdot \operatorname{By} \operatorname{Ad}\left(L^{0}\right)$-equivariance and by the fact that $\operatorname{Ad}\left(L^{0}\right)$ acts by real linear transformations, this will complete the proof of the lemma. 
Suppose first that $z=\Sigma_{\alpha \in \Delta} \xi_{\alpha} E_{-\alpha}\left(\xi_{\alpha} \in \mathbf{R}\right)$ is a regular element. We claim that the orbit of $z$ under $L^{0}$ is orthogonal to $a^{-}$. In fact, the tangents of this orbit at $z$ are of the form $[X, z](X \in \mathfrak{l})$, so it suffices to show that $\left[X, E_{-\alpha}\right] \perp E_{-\beta}$ for all $\alpha, \beta \in \Delta$. Using that $\nu E_{-\beta}=-E_{\beta}[10, \S 6]$ we have

$$
\left\langle\left[X, E_{-\alpha}\right], E_{-\beta}\right\rangle_{\nu}=\left\langle\left[X, E_{-\alpha}\right], E_{\beta}\right\rangle=\left\langle X,\left[E_{-\alpha}, E_{\beta}\right]\right\rangle
$$

which is zero by strong orthogonality, and by the fact that $\left[E_{-\alpha}, E_{\alpha}\right]=-H_{\alpha}$ $\in i \mathfrak{h}^{-} \subset q$. The tangents to the $L^{0}$-orbit and the tangents to $a^{-}$span the tangent space at $z$ in $\mathfrak{n}^{-}$by a dimension count [7, Chapter VI, Lemma 3.6]. Since $N$ is constant on $L^{0}$ orbits, our statement about the gradient follows for regular elements $z$. By continuity it is then true for all $z \in \mathfrak{a}^{-}$.

Remarks. (1) Using the Cartan decomposition as above, it is easy to show that on the tube domain $D^{c}$ the map $-r$ coincides with the symmetry of $D^{c}$ at $i E_{-\Delta}$. Since $i \Omega$ is totally geodesic in $D^{c}$, the restriction of $r$ to $i \Omega$ is the symmetry of $i \Omega$ at $i E_{-\Delta}$. It is in this context that $r$ first appeared in the early literature on tube domains.

(2) One can give another proof of Lemmas 2.3 and 2.4 with the aid of Jordan algebras. The subspace $\mathfrak{p}^{-}$has a natural Jordan algebra structure and H. Resnikoff [13] has shown that $S=\exp i n^{-}$with the exponential of the Jordan algebra. Hence, it is clear that for $z \in S$ we have $\bar{z}=z^{-1}$ (Jordan algebra inverse). The fact that $z^{-1}$ equals $1 / N(z)$ multiplied by a polynomial in the Jordan algebra is proved on p. 76 of [4]. The fact that $N$ is irreducible for irreducible $D$ is Theorem 6.2 on p. 113 of [4].

Lemma 2.5. Let $D$ be the product of the irreducible domains of tube type $D_{j}$ $(j=1,2, \ldots, p)$, and, for each $j$, let $N_{j}$ be the polynomial of Lemma 2.3 corresponding to $D_{j}$. If $M$ is a polynomial on $\mathfrak{p}^{-}$such that $|M(z)|=1$ for all $z \in S$, then

$$
M(z)=c \prod_{j=1}^{p} N_{j}(z)^{k_{j}}
$$

with $|c|=1$ and nonnegative integers $k_{j}(j=1,2, \ldots, p)$.

Proof. The proof is an argument due to Bochner [3]. By Lemma 2.4 we have, for all $z \in S$

$$
1=M(z) \overline{M(z)}=M(z) M^{\sharp}((\operatorname{grad} N(z)) / N(z))
$$

where $M^{\#}$ is the polynomial obtained from $M$ by changing all the coefficients of $M$ to their complex conjugates. Multiplying by a suitable power of $N(z)$, we have $N(z)^{k}=M(z) P(z)$ with some polynomial $P$. Since $S$ is a set of uniqueness for holomorphic functions, the above formula holds for all $z \in$ $\mathfrak{p}^{-}$. Using Lemma 2.3 to write $N(z)$ as a product of irreducible factors, the lemma follows by unique factorization. 
REMARK. For $U^{n}$ the polynomials (in fact, the entire functions) which have modulus one on the Šilov boundary were determined by Bojanic and Stoll [1]. In [3] Bochner gave a new proof of their result by a very general method which he then used to determine the polynomials which have modulus one on the Silov boundary of the domain of $n \times n$ matrices. It is Theorem 3 of the above paper of Bochner's which motivated the construction of $N$ and $r$ in this section.

3. Rational inner functions. In this section, $D$ will denote a bounded symmetric domain which is not necessarily of tube type. As pointed out in $\$ 2$, the compact group $K$ acts transitively on the Silov boundary $S$ of $D$, and $S$ is circular. Therefore, there exists on $S$ a unique normalized $K$-invariant measure $\mu$ and the following formula of Bochner [2] holds for functions $f$ : $S \rightarrow \mathrm{C}$ which are in $L^{1}$ relative to the measure $\mu$ :

$$
\int_{S} f(w) d \mu(w)=\frac{1}{2 \pi} \int_{0}^{2 \pi} \int_{S} f\left(e^{i \theta} w\right) d \mu(w) d \theta .
$$

Since $D$ is convex [12, p. 371], and therefore star-shaped relative to the origin, for every $f: D \rightarrow \mathbf{C}$ one can define for $0 \leqslant r<1$ a function $f_{r}: S \rightarrow \mathbf{C}$ by $f_{r}(w)=f(r w)$. By using (3.1) it follows easily that for $f \in H^{\infty}(D)$ (i.e., bounded and holomorphic in $D), f^{*}(w)=\lim f_{r}(w)$ exists for almost all $w \in S$, as $r \rightarrow 1$. An inner function on $D$ is a bounded holomorphic function on $D$ such that $\left|f^{*}(w)\right|=1$ for almost every $w \in S$. Since $D$ and $S$ are circular, and $D$ is star-shaped, for every $f: D \rightarrow \mathrm{C}$ and every $w \in S$, one can define a function $f_{w}: U \rightarrow \mathbf{C}$ by $f_{w}(\zeta)=f(\zeta w)\left(U=U^{1}\right.$ is the unit disc in $\left.\mathbf{C}\right)$. Let $f$ be inner in $D$, then (3.1) and Fubini's theorem immediately yield that $f_{w}$ is an inner function on $U$ for almost every $w \in S$. Another consequence of (3.1) is that for $f \in H^{\infty}(D), f^{*}$ cannot vanish on a subset of positive measure of $S$ without $f$ vanishing identically [17]. In other words, subsets of positive measure of $S$ are sets of uniqueness for $H^{\infty}$, and a fortiori for polynomials. An immediate consequence of this is the following fact which will be used repeatedly in the sequel: If $F \neq 0$ is holomophic in a neighborhood of $\bar{D}$, then the intersection of its zero set with $S$ is a closed subset of $S$ whose complement is dense and has full measure in $S$. It follows from this that subsets of positive measure of $S$ are sets of uniqueness for rational functions. Another fact which we have to use several times is the following: Let $f$ and $g$ be holomorphic in some domain $G$, such that $f / g$ is holomorphic in $G$. If the germs of $f$ and $g$ are coprime in the local ring of holomorphic functions at every $z \in G$, then $g$ does not vanish anywhere in $G$. For a proof see p. 149 of [8]. Finally, let $f=P / Q$ be a rational function on $\mathbf{C}^{n}$ in lowest terms (i.e., $P$ and $Q$ coprime), then the degree of $f$ is defined by $\operatorname{deg} f=\max \{\operatorname{deg} P$, $\operatorname{deg} Q\}$. 
LEMMA 3.1. Let $E \subset S$ be a compact uniqueness set for polynomials, and let $f$ be holomorphic in $D$.

(i) If, for every $w \in E, f_{w}$ is rational of degree $k$ and $f_{w}$ is continuous on $\bar{U}$, then there exist relatively prime polynomials $P$ and $Q$ on $\mathbf{C}^{n}$, such that $f=P / Q$, and $\operatorname{deg} f=k$.

(ii) If, in addition, $f_{w}$ is inner for every $w \in E$, then $Q$ has no zero in $D \cup E$, and $P(w)=M(w) \overline{Q(w)}$ for $w \in E$ where $M$ is $a$ unique homogeneous polynomial such that $|M|=1$ in $E$.

Proof. Assertion (i) is a special case of Theorem 5.2.2 of [14]. Assertion (ii) is a modified version of Theorem 2.2 of [15]. To prove it note first that $P$ and $Q$ being relatively prime, $Q$ has no zero in $D$. Therefore, $Q_{w}$ cannot vanish identically for any $w \in S$ and, therefore, for all $w \in E$

$$
f_{w}(\zeta)=P_{w}(\zeta) / Q_{w}(\zeta)=M(w) \zeta^{k-l} \prod_{j=1}^{l}\left(\zeta-\alpha_{j}(w)\right) / \prod_{j=1}^{l}\left(1+\overline{\alpha_{j}(w)} \zeta\right)
$$

Here $M$ and $\alpha_{j}(1 \leqslant j \leqslant l)$ are complex-valued functions in $E$ such that $|M(w)|=1$ and $\left|\alpha_{j}(w)\right|<1$. Since both sides have degree $k$, the numerators and denominators must agree separately, after we make the normalization $Q(0)=1$. Now $Q(w)=Q_{w}(1) \neq 0$ for $w \in E$, and we already know that $Q \neq 0$ on $D . M(w)$ must agree for every $w \in E$ with the highest degree term in the homogeneous expansion of $P$ and, hence, there exists a unique homogeneous polynomial (also denoted by $M$ ) whose value at every $w \in E$ is $M(w)$. Finally, set $\zeta=1$ to get

$$
P(w)=P_{w}(1)=M(w) \prod_{j=1}^{l}\left(1+\alpha_{j}(w)\right)=M(w) \overline{Q(w)}
$$

for $w \in E$.

LEMMA 3.2. If $f \in H^{\infty}(D)$ and rational of degree $k$, then $f_{w}$ is of degree $k$ for all $w$ in some dense open subset of $S$.

Proof. Let $f=P / Q$ in lowest terms. Then, as in the proof of Lemma 3.1, $f_{w}(\zeta)=P_{w}(\zeta) / Q_{w}(\zeta)$ for all $w \in S$. Regarding $P_{w}(\zeta)$ and $Q_{w}(\zeta)$ as elements of the polynomial ring $\mathbf{C}(w)[\zeta]\left(\mathbf{C}(w)\right.$ is the field of rational functions in $\left.\mathbf{C}^{n}\right)$, we cancel common factors and get $f_{w}(\zeta)=\tilde{P}_{w}(\zeta) / \tilde{Q}_{w}(\zeta)$. We can arrange that $\tilde{P}_{w}(\zeta), \tilde{Q}_{w}(\zeta) \in \mathbf{C}[w][\zeta]$, since by the Gauss lemma a factoring in $\mathbf{C}(w)[\zeta]$ implies one in $\mathbf{C}[w][\zeta]$. Now write

$$
\begin{aligned}
& \tilde{P}_{w}(\zeta)=p_{l}(w) \zeta^{l}+\cdots+p_{0}(w), \\
& \tilde{Q}_{w}(\zeta)=q_{l}(w) \zeta^{l}+\cdots+q_{0}(w)
\end{aligned}
$$

where $p_{l}(w)$ or $q_{l}(w) \neq 0$ as elements of $\mathrm{C}[w]$. The set $E_{1}=\left\{w \in S: p_{l}(w)\right.$ or $\left.q_{l}(w) \neq 0\right\}$ is dense open by a previous remark. Let $R(w)$ be the resultant of 
$\tilde{P}_{w}(\zeta)$ and $\tilde{Q}_{w}(\zeta)$. As an element of $\mathbf{C}[w], R(w)$ is nonzero, since $\tilde{P}_{w}$ and $\tilde{Q}_{w}$ have no common factor. Hence, $E_{2}=\{w \in S: R(w) \neq 0\}$ is dense open in $S$, and so is $E=E_{1} \cap E_{2}$. For fixed $w \in E$ we have $\operatorname{deg} f_{w}(\zeta)=l$, since $\tilde{P}_{w}(\zeta)$ and $\tilde{Q}_{w}(\zeta)$ have no common factor by $R(w) \neq 0$. Notice that every $f_{w}$ is continuous on $\bar{U}$ because it is bounded and rational. Therefore, by applying Lemma 3.1 to some compact subset of positive measure of $E$, it follows that $\operatorname{deg} f=l$, but we know that $\operatorname{deg} f=k$.

We can now state and prove our main result:

THEOREM 3.3. Let $f$ be a rational inner function on $D$.

(i) If $D$ is of tube type, then

$$
f(z)=M(z) Q^{\sharp}(r(z)) / Q(z),
$$

where $Q$ is a polynomial such that $Q \neq 0$ on $D$, and $r$ and $M$ are given by (2.2) and (2.3), respectively. If one normalizes $Q$ by $Q(0)=1$, then $M$ and $Q$ are uniquely determined.

(ii) If $D$ has no irreducible factor of tube type, then $f$ is constant.

(iii) If $D=D_{1} \times D_{2}$ where $D_{1}$ is of tube type and $D_{2}$ has no irreducible factor of tube type, then (with obvious notation)

$$
f\left(z_{1}, z_{2}\right)=g\left(z_{1}\right)
$$

with $g$ rational and inner on $D_{1}$.

Proof. Let $f=P / Q$ in lowest terms. Recall first that, for almost all $w \in S, f_{w}$ is inner, and that for $w$ in a dense open subset of $S, \operatorname{deg} f_{w}=\operatorname{deg} f$, by Lemma 3.2. Therefore, for $w$ belonging to the intersection $E$ of the above two sets, $f_{w}$ is a finite Blaschke product of constant degree. Exhausting $E$ by an increasing sequence of compact sets and using Lemma 3.1, it follows that $P(w)=M(w) \overline{Q(w)}$ for $w \in E$, where $M$ is a homogeneous polynomial which has modulus one on $E$, and hence by continuity on $S$. We also have $Q \neq 0$ on $D \cup E$.

In the case (i) by Lemma 1.4 we have for $w \in E$, that

$$
\overline{Q(w)}=Q^{\sharp}(w)=Q^{\sharp}((\operatorname{grad} N(w)) / N(w))
$$

and, therefore,

$$
P(w)=M(w) Q^{\sharp}((\operatorname{grad} N(w)) / N(w)),
$$

and the last equality holds everywhere in $C^{n}$ where $N \neq 0$, because $E$ is a set of uniqueness for polynomials. Now $f=P / Q$, and $M$ is of the form (2.3) by Lemma 2.5. Since $f=P / Q$ is in lowest terms, $Q$ is uniquely determined if we normalize it by $Q(0)=1$, and $M$ is unique by Lemma 3.2.

To prove (ii) we first need the following result of Schmid [16]: Let $D$ be a bounded symmetric domain which has no irreducible factors of tube type, and let $f \in L^{2}(S)$. Then $f$ is the boundary function of a function $F$ in the 
Hardy space $H^{2}$ of $D$ if and only if $f$ satisfies in the sense of distributions a finite number of differential equations $Z_{j} f=0,1 \leqslant j \leqslant \operatorname{dim} S$, where the $Z_{j}$ 's are first-order differential operators, the so-called tangential Cauchy-Riemann operators on $S$. Let now $M$ be an inner function on $D$ which is $C^{1}$ on $\bar{D}$; we claim that $M$ is constant. In fact, $Z_{j} M=0$ for all $j$, hence, for all $j$

$$
0=Z_{j} 1=Z_{j}(M \bar{M})=\left(Z_{j} M\right) \bar{M}+M\left(Z_{j} \bar{M}\right)
$$

implies that $Z_{j} \bar{M}=0$, i.e., $\bar{Z}_{j} M=0$ for all $j$. The last equations imply (by the obvious version of Schmid's theorem for antiholomorphic functions) that $\boldsymbol{M}$ is antiholomorphic. Since $M$ is both holomorphic and antiholomorphic, it is a constant. This shows that the homogeneous polynomial $M$ constructed at the beginning of the proof is constant $c$ of absolute value one. Hence, $P=c \bar{Q}$ on $S$. This implies, as above, that $P$ is simultaneously holomorphic and antiholomorphic, and hence constant. Therefore, $Q$ is constant, and so is $f=$ $P / Q$.

To prove (iii) we need the following facts from [9]. Recall that $S=S_{1} \times$ $S_{2}$. Let $f$ be a bounded holomorphic function on $D=D_{1} \times D_{2}$, then $(\alpha)$ there is a set $E$ of full measure in $S_{1}$ such that for $w_{1} \in E$

$$
f^{w_{1}}\left(z_{2}\right)=\lim _{r \rightarrow 1} f\left(r w_{1}, z_{2}\right) \quad(0 \leqslant r<1)
$$

exists uniformly for $z_{2}$ in compact subsets of $D_{2}$. This shows that $f^{w_{1}} \in$ $H^{\infty}\left(D_{2}\right)$, hence, $\left(f^{\omega_{1}}\right)^{*}$ exists as an $L^{\infty}$-function on $S_{2}$. Further: $(\beta)$ for all $w_{1} \in E$ and almost all $w_{2} \in S_{2}$

$$
\left(f^{w_{1}}\right)^{*}\left(w_{2}\right)=f^{*}\left(w_{1}, w_{2}\right) \text {. }
$$

In the present case we apply this to our inner function $f$. From $(\beta)$ we see that there is a set $E_{0} \subset E$, still of full measure, such that $f^{w_{1}}$ is inner for $w_{1} \in E_{0}$. Since $f$ is rational, by $(\alpha)$ it follows that this $f^{w_{1}}$ is also rational. $(\gamma)$ By part (ii) of this theorem $f^{w_{1}}$ is constant. $f^{w_{1}}\left(z_{2}\right)=c\left(w_{1}\right)$ for all $z_{2} \in D_{2}$, and $\left|c\left(w_{1}\right)\right|=1$. Now fix any $z_{2} \in D_{2}$ and consider the $H^{\infty}$-function $z^{2} f\left(z_{1}\right)$ $=f\left(z_{1}, z_{2}\right)$ on $D_{1}$. Let $w_{1} \in E_{0}$ and $0 \leqslant r<1$, then by $(\alpha),{ }^{z_{2}} f\left(r w_{1}\right) \rightarrow f^{w_{1}}\left(z_{2}\right)$ for $r \rightarrow 1$. By $(\gamma)$ this value equals $c\left(w_{1}\right)$. In other words: $(\delta)$ For almost all $w_{1} \in S,\left({ }^{2} f\right)^{*}\left(w_{1}\right)=c\left(w_{1}\right)$. This means that the function as an element of $L^{\infty}\left(S_{1}\right)$ coincides with $c$; hence, $\left({ }^{2} f f\right)^{*}$ is independent of $z_{2}$. Since for $h \in H^{\infty}$, $h^{*}$ determines $h$ uniquely, this implies that ${ }^{2} f$ is independent of $z_{2}$, i.e., $f\left(z_{1}, z_{2}\right)=g\left(z_{1}\right)$ for some function $g: D_{1} \rightarrow \mathbf{C}$. The function $g$ is rational and belongs to $H^{\infty}\left(D_{1}\right)$, since $f$ is such on $D$. Further, $g$ is inner, because by $(\delta)$, $g^{*}\left(w_{1}\right)=\left({ }^{2} f\right)^{*}\left(w_{1}\right)=c\left(w_{1}\right)$ for $w_{1} \in E_{0}$, and $\left|c\left(w_{1}\right)\right|=1$. Q.E.D.

REMARK. For rational inner functions on the domain $M_{n}$ of $n \times n$ matrices which are subjected to the further restriction that $f$ is continuous on $\bar{M}_{n}$, the representation formula (3.2) was obtained in [18]. 
Proposition 3.4. Let $D$ be a bounded symmetric domain.

(i) If $f$ is a continuous function on $\bar{D}$ such that $\left.f\right|_{D}$ is inner on $D$, then $f$ is rational. Further, if $f=P / Q$ in lowest terms, then $Q(z) \neq 0$ for $z \in \bar{D}$.

(ii) If $f$ is a meromorphic function on a domain containing $\bar{D}$ and $\left.f\right|_{D}$ is inner on $D$, then $f$ is rational.

Proof. The first part of the proof of (i) is an argument of Rudin [14, p. 112]. Since $f$ is continuous on $\bar{D}, f_{w}$ is inner and continuous on $\bar{U}$ for every $w \in S$ and, therefore, a finite Blaschke product. The degree of $f_{w}$ equals the index of the loop $\theta \mapsto f_{w}(\exp 2 \pi i \theta)$ in $\mathbf{C}-\{0\}$. This is a continuous integer valued function on the connected set $S$, and consequently constant. Therefore, by Lemma 3.1, $f=P / Q$ with coprime polynomials $P$ and $Q$, and $Q(z) \neq 0$ for $z \in D \cup S$. To see that $Q$ does not vanish on $\bar{D}$ note that $1 / Q$ is also holomorphic in $D$ and continuous on $D \cup S$; therefore, by [9] it extends continuously to $\bar{D}$.

REMARK. This result in conjunction with Lemma 2.5 immediately yields the known fact [3] that entire inner functions are homogeneous polynomials.

To prove assertion (ii) we begin by recalling that $D$ is convex and that, therefore, every neighborhood of $\bar{D}$ contains a dilation $(1+\varepsilon) D$ of $D$, where $\varepsilon>0$ is sufficiently small. Hence, we may, and shall, assume that the domain of $f$ is such a dilation $D^{\prime}=(1+\varepsilon) D$ of $D$. The domain $D^{\prime}$ is convex, too; therefore, it is a domain of holomorphy and its second integral cohomology group vanishes. Consequently, $f=g / h$ where $g$ and $h$ are holomorphic in $D^{\prime}$, and the germs of $f$ and $g$ are relatively prime in the local ring of holomorphic functions at every $z \in D^{\prime}$. Since $f$ is holomorphic in $D$, it follows (by a remark at the beginning of this section) that $h$ does not vanish anywhere in $D$. It follows as in the proof of Lemma 3.1 that for all $w \in S$ and all $\zeta \in U$, $f_{w}(\zeta)=g_{w}(\zeta) / h_{w}(\zeta)$. Since $g_{w}$ and $h_{w}$ can be continued analytically into $(1+\varepsilon) U$, it follows that $f_{w}$ is the restriction of a meromorphic function for all $w \in S$. By hypothesis $\left.f\right|_{D}$ is inner and, therefore, there exists a subset $S_{0}$ of $S$ which has full measure and such that for $w \in S_{0}, f_{w}$ is inner. Now it is immediate that an inner function of one variable which is the restriction to $U$ of a function meromorphic in $(1+\varepsilon) U$ is continuous on $\bar{U}$, and hence rational. Therefore, $f_{w}$ is rational for $w \in S_{0}$.

The truth of (ii) follows immediately from part (i) of Lemma 3.1 if we can prove that the degree of $f_{w}$ is constant almost everywhere on $S$. To prove that $\operatorname{deg} f_{w}$ is constant almost everywhere we need detailed information about the Taylor series of rational functions of one variable which is due to Kronecker. We state these results in the form best suited for the present purpose; their proofs are on (or readily follow from) pp. 321-323 of [5].

Let $\left\{a_{i}\right\}_{i=0}^{\infty}$ be a sequence of complex numbers. For $s, t \in \mathbf{N}(\mathbf{N}=$ natural numbers) define the Hankel determinants $D_{s}^{t}$ by 


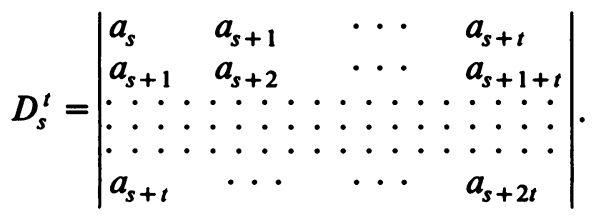

I. Let

$$
\frac{c_{0}+c_{1} \zeta+\cdots+c_{p} \zeta^{p}}{1+b_{1} \zeta+\cdots+b_{q} \zeta^{q}}=\sum_{k=0}^{\infty} a_{k} \zeta^{k}
$$

where the rational function on the L.H.S. is in lowest terms. If $t>q$, then $D_{s}^{t}=0$ for all $s \geqslant \max \{0, p-t+1\}$. Moreover, if $q>0$, then $D_{s}^{q-1} \neq 0$ for all $s>\max \{0, p-q+1\}$.

II. Conversely, let $\sum_{k=0}^{\infty} a_{k} \zeta^{k}$ be a complex formal power series. If there exists a $t \in \mathbf{N}$ such that $D_{s}^{t}=0$ for almost all (i.e., for all but finitely many) $s \in \mathbf{N}$, then the above series is the Taylor series of a rational function regular at the origin.

III. The quotient representation in lowest terms of a rational function regular at the origin can be recovered from its Taylor series as follows (we use the notation of (3.4)):

$$
q=\inf \left\{t \in \mathbf{N}: D_{s}^{t}=0 \text { for almost all } s \in \mathbf{N}\right\} .
$$

With

$$
\sigma=\inf \left\{s \in \mathbf{N}: D_{s+i}^{q}=0 \text { for all } i \in \mathbf{N}\right\}
$$

one has

$$
p \leqslant q+\sigma-1 \text { whenever } q-1+\sigma>0 .
$$

If $q=0$, then already $\sum_{k=0}^{\infty} a_{k} \zeta^{k}$ is a polynomial, and if $q=\sigma=0$, then $a_{k}=0$ for all $k \in \mathbf{N}$. If $q>0$, then the coefficients $b_{1}, \ldots, b_{q}$ can be determined uniquely from the $q \times q$ linear system

$$
\begin{aligned}
& a_{\sigma} b_{q}+a_{\sigma+1} b_{q-1}+\cdots+a_{\sigma+q-1} b_{1}+a_{\sigma+q}=0 \\
& a_{\sigma+1} b_{q}+a_{\sigma+2} b_{q-1}+\cdots+a_{\sigma+q} b_{1}+a_{\sigma+q+1}=0
\end{aligned}
$$

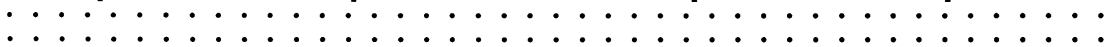

$$
\begin{aligned}
& a_{\sigma+q-1} b_{q}+\cdots+\cdots+a_{\sigma+2 q-1}=0 .
\end{aligned}
$$

Finally, the numbers $d_{j}$ defined for $j \in \mathbf{N}$ by

$$
d_{j}=\sum_{k=0}^{j} a_{k} b_{j-k}, \quad b_{0}=1 .
$$

are zero for $j \geqslant q+\sigma, p=\max \left\{j \in[0, q+\sigma-1]: d_{j} \neq 0\right\}$, and $c_{j}=d_{j}$ for $0 \leqslant j \leqslant p$. 
We can now continue with our proof. Let $f(z)=\sum_{k=0}^{\infty} a_{k}(z)$ be the homogeneous expansion of $f$, where $a_{k}$ is a homogeneous polynomial of degree $k$. This series converges absolutely and uniformly if $z$ belongs to a sufficiently small open ball $B$, centered at the origin. There exists a $\rho>0$ such that for $\zeta \in \rho U, \zeta w \in B$ for every $w \in S$. Hence for $|\zeta|<\rho$ and $w \in S$ we have

$$
f_{w}(\zeta)=f(\zeta w)=\sum_{k=0}^{\infty} a_{k}(w) \zeta^{k},
$$

and the series converges absolutely. By the uniqueness theorem of power series it is the Taylor series of $f_{w}$ (and hence converges absolutely for $\zeta \in U$ ). Denote by $D_{s}^{t}(z)$ the Hankel determinants formed with the sequence $\left\{a_{k}(z)\right\}_{k=0}^{\infty}, z \in \mathbf{C}^{n}$; they depend polynomially on $z$. For $s, t \in \mathbf{N}$ define subsets $E_{s}^{t}$ of $S$ as follows:

$$
E_{s}^{t}=\left\{w \in S: D_{s+i}^{t}(w)=0 \text { for all } i \in \mathbf{N}\right\} .
$$

These sets are (possibly empty) closed sets and $E_{s}^{t} \subset E_{s^{\prime}}^{t}$ if $s<s^{\prime}$. Since $f_{w}$ is rational for $w \in S_{0}$, by I we have that

$$
\bigcup_{s, t \in \mathrm{N}} E_{s}^{t} \supset S_{0}
$$

and, therefore, at least one of the sets $E_{s}^{t}$ has positive measure. Let

$$
\begin{gathered}
q=\inf \left\{t \in \mathbf{N}: \mu\left(E_{s}^{t}\right)>0 \text { for some } s \in \mathbf{N}\right\}, \text { and } \\
\sigma=\inf \left\{s \in \mathbf{N}: \mu\left(E_{s}^{q}\right)>0\right\} .
\end{gathered}
$$

On $E_{\sigma}^{q}$ the polynomials $D_{\sigma+i}^{q}$ vanish for all $i \in \mathbf{N}$, and hence they vanish identically. It follows that $E_{\sigma}^{q}=S$, and hence by II that $f_{w}$ is rational for every $w \in S$. If $q=\sigma=0$, then by III, $a_{k}(w)=0$ for all $w \in S$ and all $k \in \mathbf{N}$, and therefore $f$ is identically zero. From here on we exclude the case that both $q$ and $\sigma$ are zero.

Let $p_{w}$ and $q_{w}$ denote the degrees of the numerator and denominator of $f_{w}$, respectively, and define $\rho_{w}$ by

$$
\rho_{w}=\inf \left\{s \in \mathbf{N}: D_{s+i}^{q}(w)=0 \text { for all } i \in \mathbf{N}\right\} .
$$

We shall now prove that $q_{w}=q$, and that $p_{w} \leqslant q+\sigma-1$ for almost every $w \in S$. Note first that $D_{o+i}^{q}(w)=0$ for all $i \in \mathbf{N}$ and all $w \in S$ because $E_{o}^{q}=S$. Therefore by I, $q_{w} \leqslant q$ for all $w \in S$. If $q=0$, then $q_{w}=0$ for all $w \in S$. For $q>0$ we argue as follows: If $q_{w}<q$, then

$$
w \in \bigcup_{\substack{0<t<q \\ s \in \mathbf{N}}} E_{s}^{t},
$$

and therefore, on the complement $S_{1}$ of the above union we have $q_{w}=q$. But 
for $t<q$ the sets $E_{s}^{t}$ have zero measure for all $s \in \mathbf{N}$, so that $S_{1}$ has full measure. We now estimate $p_{w}$. Note that $\rho_{w} \leqslant \sigma$ for all $w \in S$, and that for $w \in S_{1}, \rho_{w}$ is equal to the number $\sigma_{w}$ defined for $f_{w}$ by (3.6). Therefore for $w \in S_{1}$ we conclude by (3.7) that $p_{w} \leqslant q_{w}+\sigma_{w}-1$, and hence that

$$
p_{w} \leqslant q+\sigma-1, \quad w \in S_{1} \text {. }
$$

Let $w \in S_{1}$. From the linear system (3.9) we determine the coefficients $b_{1}^{w}$, $b_{2}^{w}, \ldots, b_{q}^{w}$ of the denominator of $f_{w}$. Each $b_{j}^{w}$ is the quotient of two determinants whose entries are elements of the set $\left\{a_{\sigma}(w)\right.$, $\left.a_{\sigma+1}(w), \ldots, a_{\sigma+2 q-1}(w)\right\}$. (Note that the denominator is always $D_{\sigma}^{q-1}(w)$, and hence different from zero, by I.) It follows from this that $b_{j}^{w}(1 \leqslant j \leqslant q)$ is the value at $w$ of a rational function $b_{j}$ which is uniquely determined because subsets of positive measure of $S$ are sets of uniqueness for rational functions. We now form the functions $c_{j}$ defined for every $j \in \mathbf{N}$ by

$$
c_{j}(z)=\sum_{k=0}^{j} a_{k}(z) b_{j-k}(z), \quad b_{0}(z) \equiv 1 .
$$

If $w \in S_{1}$, then by III the $c_{j}(w)$ 's for $0 \leqslant j \leqslant p_{w}$ are the coefficients of the numerator of $f_{w}$, and $c_{j}(w)=0$ for $j>p_{w}$. It follows from (3.10) that $c_{j}$ is zero on $S_{1}$ for $j \geqslant q+\sigma$; therefore $c_{j}$ is identically zero for these indices. Define $p$ by $p=\max \left\{j \in[0, q+\sigma-1]: c_{j} \neq 0\right\}$, and let $S_{2}$ be the subset of full measure of $S$ on which $c_{p}(w) \neq 0$. Then for $w \in S_{1} \cap S_{2}, p_{w}=p$, and hence $\operatorname{deg} f_{w}=\max \{p, q\}$ on the set of full measure $S_{1} \cap S_{2}$. Q.E.D.

\section{REFERENCES}

1. R. Bojanic and W. Stoll, A characterization of monomials, Proc. Amer. Math. Soc. 13 (1962), 115-116.

2. S. Bochner, Classes of holomorphic functions of several variables on circular domains, Proc. Nat. Acad. Sci. U.S.A. 46 (1960), 721-723.

3. Entire functions in several variables with constant absolute values on a circular uniqueness set, Proc. Amer. Math. Soc. 13 (1962), 117-120.

4. H. Braun und M. Koecher, Jordan Algebren, Springer, Berlin, 1966.

5. P. Dienes, The Taylor series, Oxford Univ. Press, Oxford, 1931.

6. H. Freudenthal, Zur ebenen Oktavengeometrie, Indag. Math. 15 (1953), 195-200.

7. S. Helgason, Differential geometry and symmetric spaces, Academic Press, New York, 1962.

8. L. Hörmander, An introduction to complex analysis in several variables, Van Nostrand, Princeton, 1966.

9. A. Korányi, Poisson integrals and boundary components of symmetric spaces, Invent. Math. 34 (1976), 19-35.

10. A. Korányi and J. Wolf, Realization of hermitian symmetric spaces as generalized halfplanes, Ann. of Math. (2) 81 (1965), 265-288.

11. O. Loos, Symmetric spaces, Benjamin, New York, 1969.

12. C. C. Moore, Compactifications of symmetric spaces. II, Amer. J. Math. 86 (1964), 358-378.

13. H. L. Resnikoff, The maximum modulus principle for tubes over domains of positivity, Math. Ann. 156 (1964), 340-346.

14. W. Rudin, Function theory in polydiscs, Benjamin, New York, 1969. 
15. W. Rudin and E. L. Stout, Boundary properties of functions of several complex variables, J. Math. Mech. 14 (1965), 991-1006.

16. W. Schmid, Die Randwerte holomorpher Funktionen auf hermitesch symmetrischen Räumen, Invent. Math. 9 (1969), 61-80.

17. M. Stoll, Harmonic majorants for plurisubharmonic functions on bounded symmetric domains with applications to the spaces $H_{\Phi}$ and $N_{\text {, }}$, J. Reine Angew. Math. 282 (1970), 80-87.

18. K. Tsurumi and T. Kanemaru, Boundary properties of analytic functions, Sci. Rep. Tokyo Kyoiku Daigaku Sect. A 9 (1968), 199-203.

Belfer Graduate School of Science, Yeshiva Universtty, New York, New York 10033

Department of Mathematics, De Paul Universtty, Chicago, Iluinots 60614 